\title{
Anti-addiction System Development Based on Android Smartphone
}

\author{
Xiafu Pan \\ Hainan Vocational College of Political Science and Law, Haikou Hainan, 571100, China
}

Keywords: Smartphone, Android system, Anti-addiction system, Addiction index, Design

\begin{abstract}
Although the smartphone brings people more convenience and entertainment, many troubles are also caused, such as smartphone addiction problem. The smartphone gives rise to severe impacts on the life, study and work of users with poor self-control ability. Especially for those under age, smartphone addiction has become a global issue and receives extensive attention from the industry. This paper analyzes addiction index calculation method of smartphone Android system and anti-addiction system design, and displays the implementation of system functions.
\end{abstract}

\section{Introduction}

Android system is one of the most extensive and the most mature operating systems with the best stability. With strong open-source openness, Android system can provide free and complete source code downloading program for users. As well, it owns strong customization function and brings diversified and rich experience for users' different demand. Since Android system has overall structure module with good stability and uniformity, it also offers solid guarantee for good design and transplantation of anti-addiction system.

\section{Specific calculation method of smartphone addiction indexes}

Prior to anti-addiction system design for the smartphone, a set of accurate and rational addiction index calculation method should be provided. All information involved in addiction indexes should be fully quantified, which is beneficial to subsequent calculation accuracy. This paper gives the specific calculation method according to relevant concept of addiction indexes.

\section{Calculation of unit minute addiction value}

Human addiction to the smartphone is mainly judged with the time. For example, one's addiction degree in a time bucket depends on the proportion of game playing time in the total time. For the same game, the hazard degree for people is different under different situations. For instance, the game is played in the following three conditions:

Firstly, the hazard of smartphone network game is larger than that of puzzle game. Its hazard can be expressed with software hazard coefficient.

Secondly, playing game in the rest time has larger hazard than playing game in leisure time. Its hazard can be expressed with time-point hazard coefficient.

Thirdly, when one continues to plat game, the hazard in the last minute is larger than that in the first minute. Its hazard can be expressed with duration hazard coefficient.

Unit minute addiction value reflects the above three situations. It can correct the hazard of smartphone on people to every minute, and every minute corresponds to a unit minute addiction value. Besides, unit minute addiction value also indirectly embodies two indexes: addiction severity and software addiction degree. Mathematical calculation formula of unit minute addiction value is as follows:

$$
A_{t}=s_{t} \times p_{t} \times l_{t}
$$

Where, $A_{t}$ represents unit addiction value of the $\mathrm{t}^{\text {th }}$ minute in the specified time bucket; $\mathrm{s}_{\mathrm{t}}$ refers to software hazard coefficient of game software at the $\mathrm{t}^{\text {th }}$ minute; $\mathrm{p}_{\mathrm{t}}$ represents hazard coefficient at the $\mathrm{t}^{\text {th }}$ minute; $l_{\mathrm{t}}$ is duration hazard coefficient at the $\mathrm{t}^{\text {th }}$ minute. 


\section{Calculation of software easy addiction index}

Software easy addiction index is very complex. Android system platform owns numerous kinds of software, and it is very difficult for people to know which software will form software addiction. For instance, if one's WeChat operation duration is $60 \mathrm{~min}$, unit minute addiction value within the $60 \mathrm{~min}$ will accumulate continuously and finally form WeChat addiction accumulative value. At present, WeChat addiction has no contingency. WeChat has becomes a kind of major software which the majority of people will be addicted to. Thus, this paper calculates software easy addiction indexes based on moving average method.

The thought of moving average method is to predict and calculate future information through the latest group of measured data. Such method can furthest reduce random fluctuation generated in the prediction process. Since the recent data are adopted, the reference and correlation are also guaranteed. This paper applies this method to achieve linear increase judgment method of different data and different weight. So, easiest addiction software A is taken for example. Its easy addiction index $\mathrm{E}_{\mathrm{A}}$ should be:

$$
E_{A}=\left(w_{1} B_{A 1}+w_{2} B_{A 2}+\ldots+w_{n} B_{A n}\right) /\left(w_{1}+w_{2}+\ldots+w_{n}\right)
$$

Where, $n$ means that the data range is the past $n$ days; $B_{S n}$ is accumulative addiction value of Software A $\mathrm{n}$ days ago; $\mathrm{w}_{\mathrm{n}}$ refers to relevancy weight value $\mathrm{n}$ days ago. When $\mathrm{n}$ is closer, its relevancy weight value is larger. To calculate addiction indexes, accumulative addiction value $\mathrm{B}_{\mathrm{S}}$ of the software should be settled once per day, and new data should be added continuously to ensure accuracy and instantaneity of addiction indexes.

\section{Calculation of addiction severity index}

Addiction severity index is calculated on the basis of software easy addiction index. Addiction severity index weighs addiction dependence degree of smartphone users on a kind of software. It completely starts from users, divides addiction severity for users and then divides anti-addiction handling grade according to corresponding grade. Here, we assume addiction severity index is X, each unit minute addiction value within the specified time is superposed to gain

$$
X=\sum_{t=1}^{n} A_{t}=\sum_{t=1}^{n} s_{t} \times p_{t} \times e^{0.0155 d_{t}-0.1505}
$$

Where, $\mathrm{n}$ represents the total time consumed of each kind of smartphone software within a time bucket in one day, and the unit is minute ${ }^{[1]}$.

\section{Anti-addiction system design}

\section{Analysis of overall system architecture}

Anti-addiction system of Android smartphone includes application layer and framework layer. This system involves application development, modification and expansion etc. the application layer can achieve basic functions of anti-addiction. It is actually divided into anti-addiction management and anti-addiction service. Anti-addiction management contains user interactive Activity interface through which smartphone users can check anti-addiction information of each kind of software and modify anti-addiction standard anytime. Anti-addiction service achieves backstage service through Android Service module which monitors operating state of each kind of Smartphone software in all weathers, owns the functions of monitoring user-defined broadcast and specific broadcast and can handle most addiction inspection tasks.

The framework layer is a main core part of anti-addiction system function extension. It is responsible for operating remote monitoring management module and protection module, protecting and modifying the original interface in Android system framework layer so as to make sure anti-addiction application program operates safely and stably. When any abnormity occurs in anti-addiction application operation program, the framework layer will start self-recovery function, 
inform remote supervisor to call SMS sending and receiving port and finally reach the effect of remote monitoring ad smartphone control.

\section{Analysis of framework layer extension function}

Framework layer extension of Android system involves modification and extension which are also the difficulties in development and design of anti-addiction system. The design difficulty is caused by complexity of Android system framework layer. This paper displays mutual relations of modification and extension modules in structure composition in the framework layer through the structure chart, and the relationship between them and external role, as shown in Fig.1.

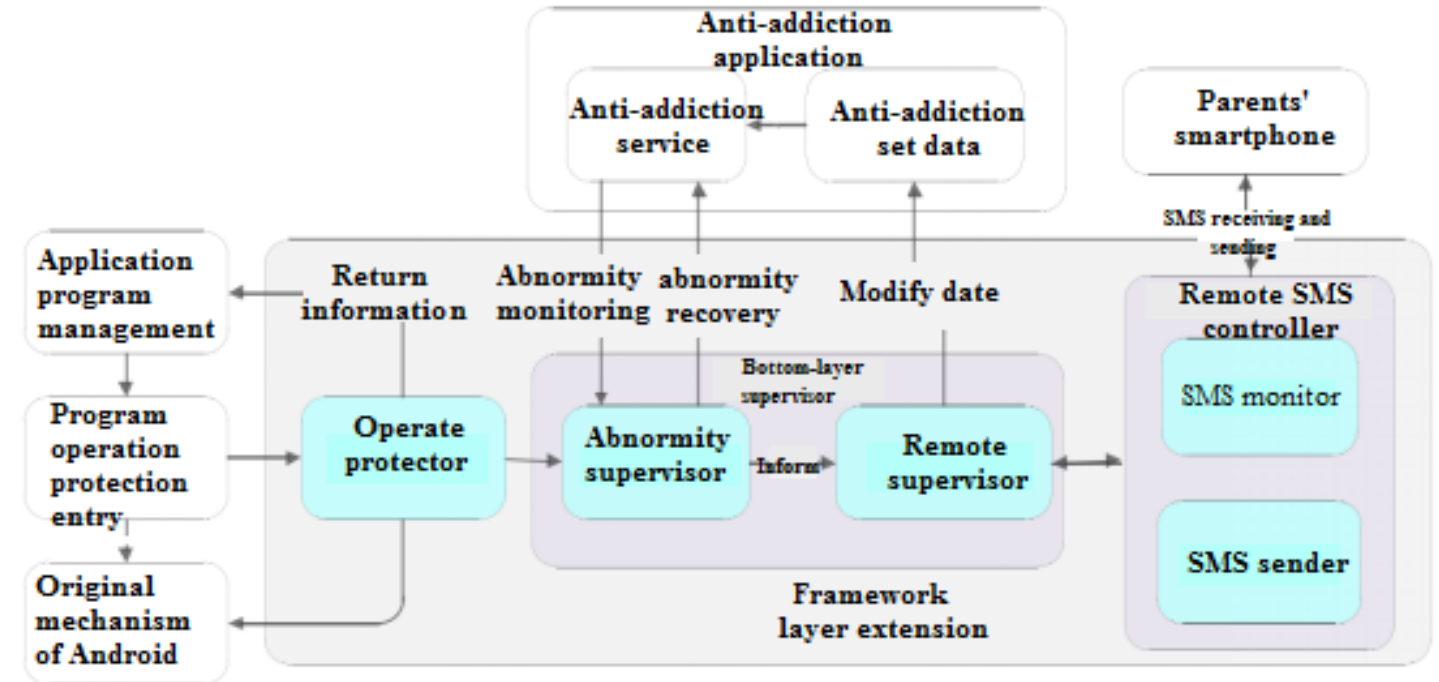

Fig.1. Schematic diagram of framework layer extension relation in Android anti-addiction system

As shown in Fig.1, framework layer extension module is in the anti-addiction system of Android system, and it includes three parts: operation protection, bottom-layer supervision and remote SMS control. Bottom-layer supervision also contains remote supervision and abnormal supervision, while remote SMS control involves two links: SMS monitoring and sending. Next, this paper mainly introduces three components of framework layer extension module.

Operation protection

Operation protection is the first barrier of framework layer for anti-addiction in the application layer, smartphone users may use task manager to operate it as required by the system or apply third-party smartphone steward software for operation protection. Such operation can intervene in normal operation of anti-addiction application. For instance, it can achieve forced closure and deletion of data, even force to unload some inherent smartphone software and realize forced prohibition of operation.

\section{Bottom-layer supervision}

It is the kernel module of framework layer extension intensification function. It can achieve abnormal supervision and remote supervision. The original intention of abnormity supervision design is to supplement the functions of operation protection module, but in fact its protection thought is different from operation protection. It is not beforehand protection, but post detection protection. It can automatically achieve system abnormity remediation and recovery. When recovery fails, it will immediately inform remote supervisor, which is called "parent informing function" of anti-addiction system. Meanwhile, it can receive remote control information send by remote monitoring system, then modify some anti-addiction management preset data according to information content and achieve complete remote smartphone management function.

\section{Remote SMS control}

Remote SMS control is specially used to achieve remote supervision. It contains SMS monitoring and SMS sending and achieves remote control and remote monitoring of Android system. To meet users' different demand, it also sets prior monitoring level, classifies different SNS, achieves analysis 
of SMS contents from diverse perspectives and finally informs remote supervision function to execute corresponding instructions, such as SMS interception.

\section{Database design analysis}

To design Android system database, SQLite database with good compatibility may be adopted. It can realize local preservation and management of data, weighs redundant information in the database as needed, improve database operation efficiency and finally achieve database design function. Database design mainly includes the following 4 parts.

Firstly, application information table can record basic information of installed software in Android system, including recording attribute information about anti-addiction system. It is also deemed as the fundamental and core data standard of anti-addiction system.

Secondly, duration management table manages data manually. Its anti-addiction system is directly installed in data management software under parent function and can achieve digital judgment of addiction standards. In other words, duration management table can save all index data set in the supervision module.

Thirdly, operation record sheet can operate an application in a time bucket, manage addiction accumulation degree, software easy addiction index and addiction severity index, and offer data calculation for the three indexes.

Fourthly, system log sheet is mainly responsible for saving all system events in the system, such as inspection of a time point. If severe addiction is discovered, addiction handling should continue to be executed until abnormal behavior is recovered. It presents intelligence, and humanization idea, and it is the most important backstage technology support in anti-addiction system ${ }^{[2]}$.

\section{Analysis of application and implementation of anti-addiction system}

\section{Environment setup implementation of anti-addiction system}

Environment setup implementation of anti-addiction system of Android smartphone mainly experiences 4 steps.

Step 1, Android source code downloading. Source code downloading should be conducted according to experiment platform and corresponding version. For example, on Google nexus platform, protogenetic Android system offered officially should be downloaded. Other smartphones should select corresponding source code provided by third-party ROM.

Step 2, source code compilation operation. Before the source code is compiled, corresponding compilation tool and dependence package should be downloaded first. In the compilation process, it is necessary to take into account of potential errors, such as whether the compilation tool and dependence package are consistent with source code version used by current Android.

In source code compilation process, make instruction should be applied to implement global compilation. Then, mmm instruction should be applied to implement module compilation. For instance, when Activity Manager Service path is modified in the system, the new path is updated in the new system after the modification to achieve the purpose of system modification and customization.

Step 3, Eclipse importing debugging. Because Android source code engineering compilation is quite complex and huge, uniform management software of a code should be prepared, such as Source Insight code reader. This code reader owns Eclipse system which supports corresponding Android system. After Android system is finely turned, Android source code can be completely imported in Eclipse. After this operation, complex source code reading efficiency of the system will improve a lot.

Step 4, smartphone refreshing. The modified and extended system is refreshed in smartphone system to detect actual application feasibility and security of anti-addiction system. It contains the influence of anti-addiction system on smartphone property and power consumption, and whether remote monitoring function can operate normally. After all relevant source codes are compiled, they 
generate refreshing zip package mode. Refreshing is carried out through card refreshing or machine refreshing ${ }^{[3]}$.

\section{Summary}

This paper discusses the hot issue - smartphone addiction from specific calculation of anti-addiction index and anti-addiction system design. Anti-addiction system design based on Android smartphone can only prohibit addiction behavior objectively and play the assistant management role. More importantly, smartphone users should enhance their subjective consciousness and establish correct value attitude to the use of smartphone software in order to fundamentally solve smartphone addiction problem.

\section{Acknowledgement}

This paper belongs to the topic of Hainan Natural Science Funds in 2015, No.: 20156244

\section{References}

[1] Wang Lu, Study on Application of Anti-addiction System Based on Distributed Database. Electronic Testing, 2014(16):48-49.

[2] Zhu Ruolei, Study on Anti-addiction System with Kernel Mode Injection Technology. Journal of Southwest China Normal University (Natural Science Edition), 2011,36(4):147-152.

[3] Zhou Ruijing, Research and Development of Android Smartphone Anti-addiction System. Sun Yat-Sen University, 2014,29-50. 